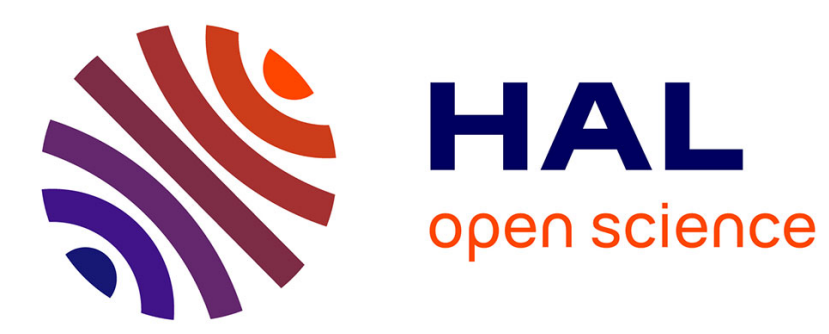

\title{
A Computational Framework for Prime Implicants Identification in Noncoherent Dynamic Systems
}

Francesco Di Maio, Samuele Baronchelli, Enrico Zio

\section{To cite this version:}

Francesco Di Maio, Samuele Baronchelli, Enrico Zio. A Computational Framework for Prime Implicants Identification in Noncoherent Dynamic Systems. Risk Analysis, 2015, 35 (1), pp.142-156. 10.1111/risa.12251 . hal-01177008

\section{HAL Id: hal-01177008 \\ https://hal.science/hal-01177008}

Submitted on 16 Jul 2015

HAL is a multi-disciplinary open access archive for the deposit and dissemination of scientific research documents, whether they are published or not. The documents may come from teaching and research institutions in France or abroad, or from public or private research centers.
L'archive ouverte pluridisciplinaire HAL, est destinée au dépôt et à la diffusion de documents scientifiques de niveau recherche, publiés ou non, émanant des établissements d'enseignement et de recherche français ou étrangers, des laboratoires publics ou privés. 


\title{
A COMPUTATIONAL FRAMEWORK FOR PRIME IMPLICANTS IDENTIFICATION IN NON-COHERENT DYNAMIC SYSTEMS
}

\author{
Francesco Di Maio ${ }^{1}$, Samuele Baronchelli ${ }^{1}$, Enrico Zio ${ }^{1,2}$ \\ ${ }^{1}$ Energy Department, Politecnico di Milano \\ Via Ponzio 34/3, 20133 Milano, Italy \\ francesco.dimaio@polimi.it \\ ${ }^{2}$ Chair on System Science and Energetic Challenge \\ European Foundation for New Energy - Electricite de France \\ Ecole Centrale, Paris, and Supelec, Paris, France
}

\begin{abstract}
Dynamic reliability methods aim at complementing the capability of traditional static approaches (e.g., Event Trees (ETS) and Fault Trees (FTs)) by accounting for the system dynamic behavior and its interactions with the system state transition process. For this, the system dynamics is here described by a time-dependent model that includes the dependencies with the stochastic transition events. In this paper, we present a novel computational framework for dynamic reliability analysis whose objectives are i) accounting for discrete stochastic transition events and ii) identifying the prime implicants (PIs) of the dynamic system. The framework entails adopting a Multiple-Valued Logic (MVL) to consider stochastic transitions at discretized times. Then, PIs are originally identified by a Differential Evolution (DE) algorithm that looks for the optimal MVL solution of a covering problem formulated for MVL accident scenarios. For testing the feasibility of the framework, a dynamic non-coherent system composed by five components that can fail at discretized times has been analyzed, showing the applicability of the framework to practical cases.
\end{abstract}

Keywords: Dynamic reliability, Prime Implicants, Multiple-Valued Logic, Differential Evolution.

\section{INTRODUCTION}

Probabilistic Risk Assessment (PRA) aims at capturing the scenarios of failure that can affect the system, and quantifying the likelihood of their occurrence and the consequences they may produce [Stuk, 1993]. The assessment is based on static system modeling tools, such as Fault Trees (FTs) and Event Trees (ETs), which allow describing the logic of system failure and accident evolution, and quantifying the related probabilities [Rosenberg, 1996; Zio, 2007]. However, FTs and ETs cannot adequately account for the impact of the dynamic interactions among continuous physical 
parameters of the process (temperature, pressure, speed, etc.), stochastic discrete failure events of the hardware and software components, and human operators actions dynamic systems [Marseguerra et al., 1996; Devooght, 1997; Pate'-Cornell, 2002; Li et al., 2005; Kirschenbaum et al., 2009; Aldemir, 2013]. PRA of dynamic systems calls also accounting for sequence and timing of the events, because these influence the development of the accident scenario, and Minimal Cut Sets (MCS), i.e., the minimal combination of elementary events that make the whole system fail within a static reliability analysis [Quine, 1952], lose their meaning [Morreale, 1967]. In these cases, PIs have been introduced as an extension of MCSs to convey the information on the minimum combinations of failures (with a certain order and timing) that lead the system to failure and that cannot be covered by more general implicants [Garret et al., 1999]. Moreover, the logic behind these dynamic interactions can give rise to non-coherent structure functions, where both failed and working states of the same components can lead the system to failure. As an example of non-coherent system, suppose that a system is composed by the components $J, K$ and $L$ and that the combination of events $\bar{J}, \bar{K}, L$ (components $J$ and $K$ failed and component $L$ working) causes a catastrophic system failure, i.e., the system has a non-coherent structure function. PIs identification can help developing an effective maintenance schedule for non-coherent systems: if components $J$, $K$ and $L$ have failed, $L$ should be the last component to be repaired in order to avoid system failure and additional counteracting measures could be taken to prevent system failure, for example by forcing failure of component $L$ when component $J$ and $K$ have already failed [Sharvia et al., 2008]. Moreover, a realistic reliability analysis should consider failures that can occur at any continuous time, with any order and magnitude: when component failures occur at different times and/or with different magnitudes, the same combination of failed components does not lead unequivocally to one failure mode and the same scenario can result in different failure modes [Devooght, 1992]. Therefore, the so-called dynamic reliability methods have been developed aiming at explicitly modeling these interaction phenomena [Devooght, 1997]. Dynamic reliability methods can be categorized into continuous and discrete approaches [Aldemir, 2013]: the former entail, at each point of time, to model the stochastic variability of accident scenarios evolution, whereas the latter consider discrete times of failure events.

Continuous Event Trees (CETs) [Devooght et al., 1992; Kopustinskas et al., 2005] provide a realistic framework for all possible failure events due to process/hardware/software/firmware/ human interactions into a single integrated model. However, the application of this and other continuous time techniques is limited by their computationally intensive nature and by the need of tailoring their algorithms to the system under consideration. 
The conceptually simpler discrete time approach by direct Monte Carlo Simulation (MC) [Marseguerra et al., 1996; Labeau, 2006] generates most probable time branchings for the ET to simulate a discrete set of accident scenarios. Dynamic Event Trees (DETs) [Hofer et al., 2004; Hakobyan et al., 2008] determine the system responses to different accident events timings and sequences by branching conditions selected by the analyst. The major challenge of DETs is the need of processing a massive amount of data for any single initiating event considered and, thus, it requires an efficient post-processing tool for treating the accumulated information [Di Maio et al., 2011a]. Petri Nets allow dynamic reliability analysis by modeling the system in terms of information transmission between nodes of a graph structure [Petersen, 1981]: nodes represent a set of all possible system states, links are possible state transitions and an initial token placed into one of the nodes represents the initial system state condition at the initial time. Discrete state simulations are run moving tokens among places, recording system state changes and treating parameter evolution [Labeau et al., 2000]. Dynamic Flowgraph Methodology (DFM) provides a modeling and analysis environment in which the system variables are represented by a finite number of states and the system dynamics is expressed by a cause-and-effect relationship between these states [Aldemir, 2013]. DFM is based on parameter values discretization and can produce "timed FTs", which are FTs in which timing relations are systematically taken into account [Garret et al., 1999]. The FTs can then be analyzed to determine how the system can reach a certain failure mode by backtracking faults by deductive/inductive analysis [Aldemir, 2013], yielding the Prime Implicants (PIs), i.e., the minimal sets of component failure events that are sufficient to cause a failure (top event) of a dynamic system and that cannot be covered by a more general implicant [Quine, 1952].

The objective of this work is to develop a discrete time framework for the dynamic reliability analysis of non-coherent systems, which i) discretizes continuous variables ii) accounts for stochastic discrete events iii) identifies the prime implicants (PIs) of a dynamic system, where the time of occurrence of each component failure is important for the determination of the system parameters evolution. We use Multiple-Valued Logic (MVL) theory for accurately modeling the behavior of the system, where timing and sequences of component failure events can be critical in determining the system failure mode. In fact, MVL theory increases the limited description capability of binary variables in real cases [Garibba et al., 1985], and components can thus be in different states of operation (for example, a valve that can be closed, partially closed or fully open, or can fail at different times). For ease of illustration of the continuous to discrete time representation proposed, we resort to a case study composed by five components that can fail at different discrete times with random magnitudes, where the time of occurrence of each component 
failure is important for the determination of the system parameters evolution and its failure mode. These simulated accident scenarios aim at reproducing real conditions that can be envisaged in real complex systems, as in the Residual Heat Removal system (RHRs) in the High Temperature-Pebble Modular Reactor (HTR-PM) [Zio et al., 2010; Di Maio et al., 2011b], where specific components failures influence the outlet water temperature.

PIs identification is here tackled as an optimization problem, which is a Set Covering Problem (SCP) [Beasley et al., 1996] defined by the MVL accident scenarios, whose solution amounts to finding the minimum combination of implicants that can guarantee the best coverage of all the minterms that make the system fail. The difficulty in developing efficient methods for PIs identification lies in the fact that each subset of implicants has an associated cost proportional to its dimension and the objective of the problem is to choose the smallest group of subsets whose union contains the whole set with minimal cost (this will be explained in details in what follows).To overcome this hurdle, the optimization problem for PIs identification is here solved in an original way with a short computational time, by a Differential Evolution (DE)-based algorithm [Storn et al., 1996] previously applied for the identification of MCSs [Di Maio et al., 2013].

The paper is organized as follows. In Section 2, the case study used to generate the accident scenarios for the dynamic reliability analysis is presented, its non-coherence is shown and the limitation of a static reliability analysis is highlighted in that it may lead to a conservative PIs identification. In Section 3, MVL theory is introduced with reference to the case study considered. The DE-based approach is presented is Section 4 and, then, applied for PIs identification in the dynamic case study of Section 5. Conclusions and remarks are given in Section 5.

\section{CASE STUDY}

\subsection{Static reliability analysis}

In order to show the way of general applicability of the proposed framework of PIs identification for dynamic and non-coherent systems, we consider a system composed by 5 components $(A, B, C$, $D$ and $E$ ), that can fail at continuous random times with random magnitudes, giving rise to different scenarios, e.g., like those plotted in Fig. 1 with reference to a safety-relevant signal (continuous line) which needs to remain bounded within pre-defined thresholds for safety (dotted and dashed lines) [Baraldi et al., 2012]. The system life is set to $T=7$ [h]. If the safety-relevant signal exceeds the upper threshold value of 2.5 , the system fails in the "High" failure mode; if it decreases below the lower threshold value of -1.5 , the system failure mode is "Low". 


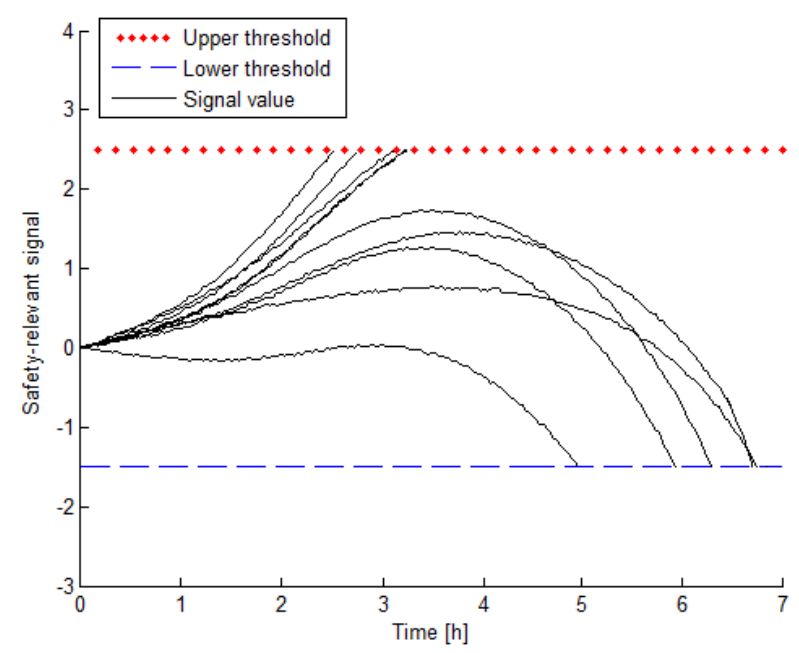

Fig 1. Evolution of the safety-relevant signal during simulated accident scenarios

For example, the average temperature of the diathermic oil of the secondary loop of a Lead Bismuth Eutectic eXperimental Accelerator Driven System (LBE-XADS) can be considered as safetyrelevant signal: if it goes beyond the upper threshold of $340{ }^{\circ} \mathrm{C}$, oil physical and chemical properties could be degraded, whereas if it goes below the lower threshold of $280{ }^{\circ} \mathrm{C}$ the structural components of the reactor could suffer of thermal shock [Zio et al., 2012]. Other examples of physical thresholds are: the upper limit of $1600{ }^{\circ} \mathrm{C}$ for the fuel cladding temperature of the High Temperature-Pebble Modular Reactor (HTR-PM), that allows the safety function of fission products retain [Di Maio et al., 2011b] or the lower limit of the water level of a pressurizer in Pressurized Water Reactor (PWR), that avoids the undesirable state of uncovered electric heaters [Baraldi et al., 2013].

A real system which has a strong similarity with the proposed case is the Residual Heat Removal system (RHRs) of the HTR-PM whose function is removing the residual heat from the core after a reactor shut-down [Di Maio et al., 2011b]. This safety system is composed of two circuits (trains) dedicated to heat removal, each one being connected to a loop of the primary circuit. The function of the RHRs trains is to transfer heat by natural convection from the reactor vessel through the water cooling wall and pipes to the air-cooling heat exchanger located in the air-cooling tower. The outlet temperature in each one of the two trains represents the equivalent of our safety-relevant signal. In fact, the outlet temperature cannot exceed $126{ }^{\circ} \mathrm{C}$ (upper threshold) because otherwise its thermal transmission properties would deteriorate and drive the system into the "High" failure mode. On the other hand, a temperature value equal to that of the cold sink of the air-water exchanger in the air cooling tower (lower threshold) would put the train out of service into the "Low" failure mode, and, therefore, cause a sharp increase in the outlet temperature of the other train [Di Maio et al., 2011b]. 
The basic failure events which can produce an increase of the outlet temperature can be: the reduction of the number of air cooling pipes in the two air-water heat exchangers (e.g. failure of components $A$ and $B$ ), the reduction of the number of water cooling wall pipes connecting the two RHRs trains with the primary circuit (e.g., failure of component E), whereas the temperature decrease can be caused by the blockage of the inlet shutters of the air cooling tower (e.g. failure of components $C$ and $D$ ) [Di Maio et al., 2011b].

In our case study, a Monte Carlo sampling procedure injects faults of random magnitudes at $t=0$ for all possible combinations of components failures (Tab. 1). The number of components that fail in an accident scenario is sampled from a binomial distribution with parameters $n=5$ (the number of components) and $p=0.8$ (so that even rare multiple fault events are included in the set of accident scenarios simulated [Zio et al., 2009]).

The equations used to simulate the safety-relevant signal evolution $y(t)$ during each of the system configurations of Tab. 1 are shown in Tab. 2. Parameters $a, b, c, d, \omega, \alpha_{1}, \alpha_{2}$ and $\alpha_{3}$ are randomly sampled from the distributions listed in Tab. 3 and can be interpreted as determining the magnitude of the failure events of the components $A, B, C, D$ and $E$. They are obtained by stratified sampling [Di Maio et al., 2011a].

\begin{tabular}{|c|c|c|}
\hline $\begin{array}{c}\text { System } \\
\text { configuration }\end{array}$ & Components that do not fail & Components that fail \\
\hline 1 & $A, B, C, D$ and $E$ & - \\
\hline 2 & $B, C, D$ and $E$ & $A$ \\
\hline 3 & $A, C, D$ and $E$ & $B$ \\
\hline 4 & $A, B, D$ and $E$ & $C$ \\
\hline 5 & $A, B, C$ and $E$ & $D$ \\
\hline 6 & $A, B, C$ and $D$ & $E$ \\
\hline 7 & $C, D$ and $E$ & $A$ and $B$ \\
\hline 8 & $B, D$ and $E$ & $A$ and $C$ \\
\hline 9 & $B, C$ and $E$ & $A$ and $D$ \\
\hline 10 & $B, C$ and $D$ & $A$ and $E$ \\
\hline 11 & $A, D$ and $E$ & $B$ and $C$ \\
\hline 12 & $A, C$ and $E$ & $B$ and $D$ \\
\hline 13 & $A, C$ and $D$ & $B$ and $E$ \\
\hline 14 & $A, B$ and $E$ & $C$ and $D$ \\
\hline 15 & $A, B$ and $D$ & $C$ and $E$ \\
\hline 16 & $A, B$ and $C$ & $D$ and $E$ \\
\hline 17 & $D$ and $E$ & $A, B$ and $C$ \\
\hline 18 & $C$ and $E$ & $A, B$ and $D$ \\
\hline 19 & $C$ and $D$ & $A, B$ and $E$ \\
\hline 20 & $B$ and $E$ & $A, C$ and $D$ \\
\hline 21 & $B$ and $D$ & $A, C$ and $E$ \\
\hline 22 & $B$ and $C$ & $A, D$ and $E$ \\
\hline
\end{tabular}




\begin{tabular}{|c|c|c|}
\hline $\mathbf{2 3}$ & $A$ and $E$ & $B, C$ and $D$ \\
\hline $\mathbf{2 4}$ & $A$ and $D$ & $B, C$ and $E$ \\
\hline $\mathbf{2 5}$ & $A$ and $C$ & $B, D$ and $E$ \\
\hline $\mathbf{2 6}$ & $A$ and $B$ & $C, D$ and $E$ \\
\hline $\mathbf{2 7}$ & $E$ & $A, B, C$ and $D$ \\
\hline $\mathbf{2 8}$ & $D$ & $A, B, C$ and $E$ \\
\hline $\mathbf{2 9}$ & $C$ & $A, B, D$ and $E$ \\
\hline $\mathbf{3 0}$ & $B$ & $A, C, D$ and $E$ \\
\hline $\mathbf{3 1}$ & $A$ & $B, C, D$ and $E$ \\
\hline $\mathbf{3 2}$ & - & $A, B, C, D$ and $E$ \\
\hline
\end{tabular}

Tab. 1. Possible system configurations for the case study

\begin{tabular}{|c|c|}
\hline Failed Component & Safety-relevant signal \\
\hline$A$ & $y(t)=2 \alpha_{1} a\left[1+e r f\left(\frac{t}{\sqrt{2}}\right)\right]+10^{-3 \omega}$ \\
\hline$B$ & $y(t)=2 \alpha_{1} a\left[1+\operatorname{erf}\left(\frac{t}{\sqrt{2}}\right)\right]+10^{-3 \omega}$ \\
\hline$C$ & $y(t)=-\alpha_{2}\left(c^{d^{t}}-c\right)+10^{-3 \omega}$ \\
\hline$D$ & $y(t)=-\alpha_{2}\left(c^{d^{t}}-c\right)+10^{-3 \omega}$ \\
\hline$E$ & $y(t)=\alpha_{3} b t+10^{-3 \omega}$ \\
\hline
\end{tabular}

Tab. 2. Equations used to simulate the safety-relevant signal evolution for each failed component

\begin{tabular}{|c|c|c|c|}
\hline Parameter & Distribution & Mean value & Standard deviation \\
\hline$a$ & Gaussian & 0.4 & 0.017 \\
\hline$b$ & Gaussian & 0.4 & 0.017 \\
\hline$c$ & Gaussian & 1.3 & 0.033 \\
\hline$d$ & Gaussian & 1.3 & 0.017 \\
\hline$\alpha_{1}$ & Gaussian & 1 & 0.083 \\
\hline$\alpha_{2}$ & Gaussian & 1.05 & 0.033 \\
\hline$\alpha_{3}$ & Gaussian & 1 & 0.033 \\
\hline$\omega$ & Gaussian & 0 & 1 \\
\hline
\end{tabular}

Tab. 3. Distribution of the parameters of the Equations of Tab. 2

Without loss of generality, among the system configurations of Tab. 1 (also referred to as minterms), we search for those leading to the failure mode "Low". PIs are those minterms whose set of variables (that in this case are Boolean and indicate if at $t=0$ components $A, B, C, D$ or $E$ are 
failed $(X)$ or not $(\bar{X})$ ), represent minimal accident component failures necessary for the failure mode occurrence and cannot be covered by a more reduced implicant [Quine, 1952]. The PIs identification is performed in an original way with a DE-based algorithm (see Section 4), which has been proven to provide a faster search than Quine-McCluskey algorithm [Di Maio et al., 2013]. As we shall see, DE is fed with a set of $N P$ chromosomes $\bar{x}=\left(x_{1}, x_{2}, \ldots, x_{R}\right)$, each one representing a possible solution made of $R$ Boolean implicant values $x_{i}$, equal to 1 if the $i$-th Boolean implicant is considered in the solution, otherwise 0 .

The 7 PIs, all of third-order (i.e., three components are listed for each implicant) are listed in Tab. 4. Notice the non-coherence of the system, as both failed and safe components contribute to the system failure mode.

\begin{tabular}{|c|}
\hline Prime Implicants (static reliability analysis) \\
\hline \\
$|\bar{A}| C|\bar{E}|$ \\
$|\bar{B}| C|\bar{E}|$ \\
$|\bar{A}| D|\bar{E}|$ \\
$|\bar{B}| D|\bar{E}|$ \\
$|C| D|\bar{E}|$ \\
$|\bar{B}| C|D|$ \\
$|\bar{A}| C|D|$ \\
\hline
\end{tabular}

Tab. 4. PIs of the "Low" failure mode in the static reliability analysis

\subsection{Dynamic reliability analysis}

The assumption that all components that fail in a scenario do so all at the same time $t=0$ is unrealistically conservative. In a realistic (but computationally intractable) analysis one should consider failures that can occur at any continuous time, with any order and magnitude [Devooght, 1992]. As a tractable approximation, we propose a computational framework that assumes that the components can fail at discrete times, e.g. values: $t=0[\mathrm{~h}], t=2[\mathrm{~h}]$ and $t=5[\mathrm{~h}]$ for our case study, representative of "early", "intermediate" and "late" failures, respectively, rather than only at $t=0$ as in the previous analysis of Section 2.1.

With these settings, it can be concluded that the same combination of failed components does not lead unequivocally to one failure mode: when component failures occur at different times and/or with different magnitudes, the resulting failure mode can be different. For example, if a failure 
occurs "late" in the mission time (as opposed to an "early" failure event), it may not lead to system failure or viceversa (if the system is non-coherent) [Di Maio et al., 2011a]. This is shown in Fig. 2 where the frequencies of the three system failure modes ("High", "Safe" and "Low") are plotted for the 32 system configurations of Tab. 1, when times of failures are sampled from discrete distributions and failure magnitudes from continuous distributions.

In Fig. 3 the same plot for the frequencies of system failure modes are reported for system configurations generated with continuous random times and magnitudes. It can be seen from the similarity of the histograms of Fig. 2 and Fig. 3 that the hypothesis of discrete (early, intermediate and late) failure times is a good approximation.

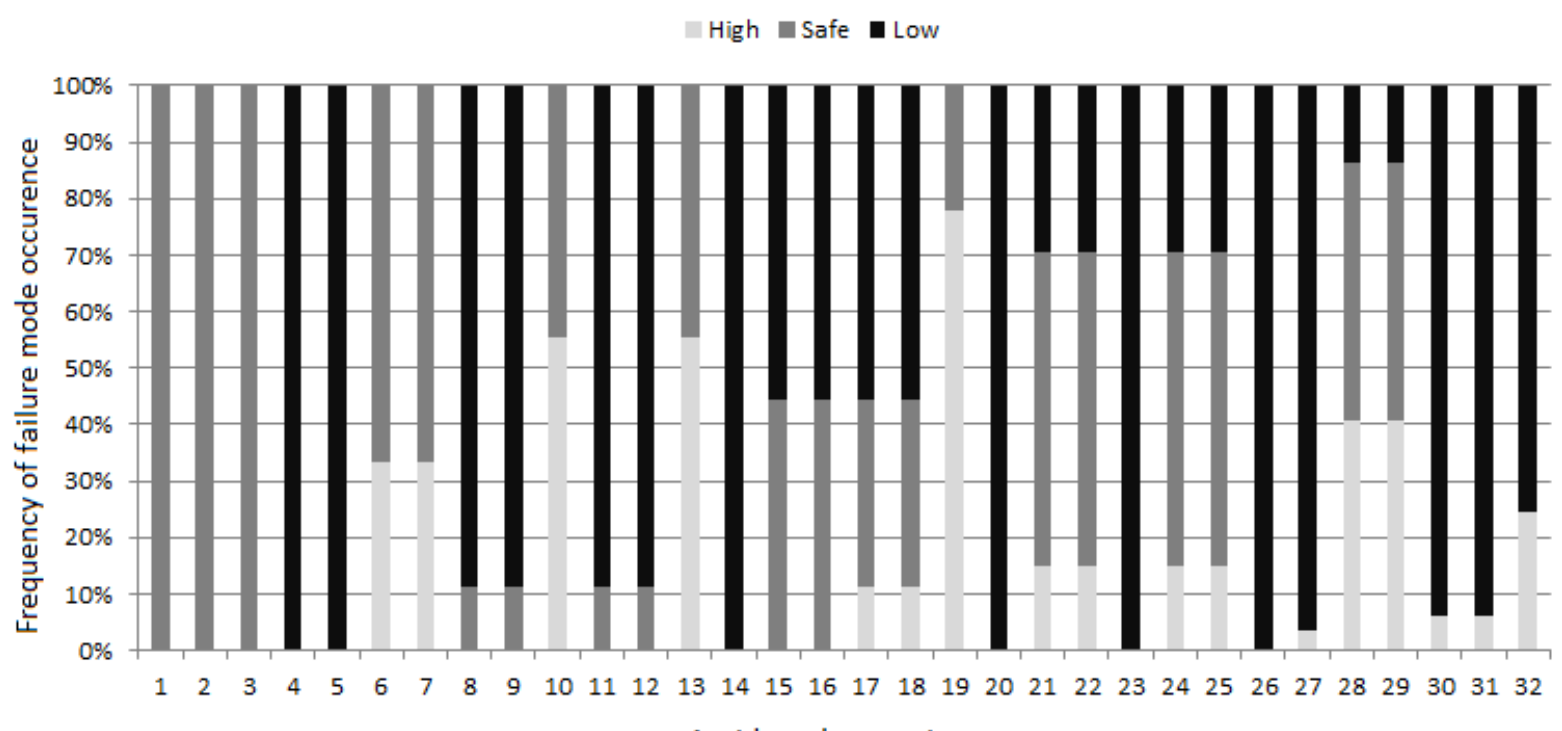

Fig. 2. Histograms of the frequency of the failure modes for each of the 32 system configurations of Tab. 1, simulated with discrete failure times 


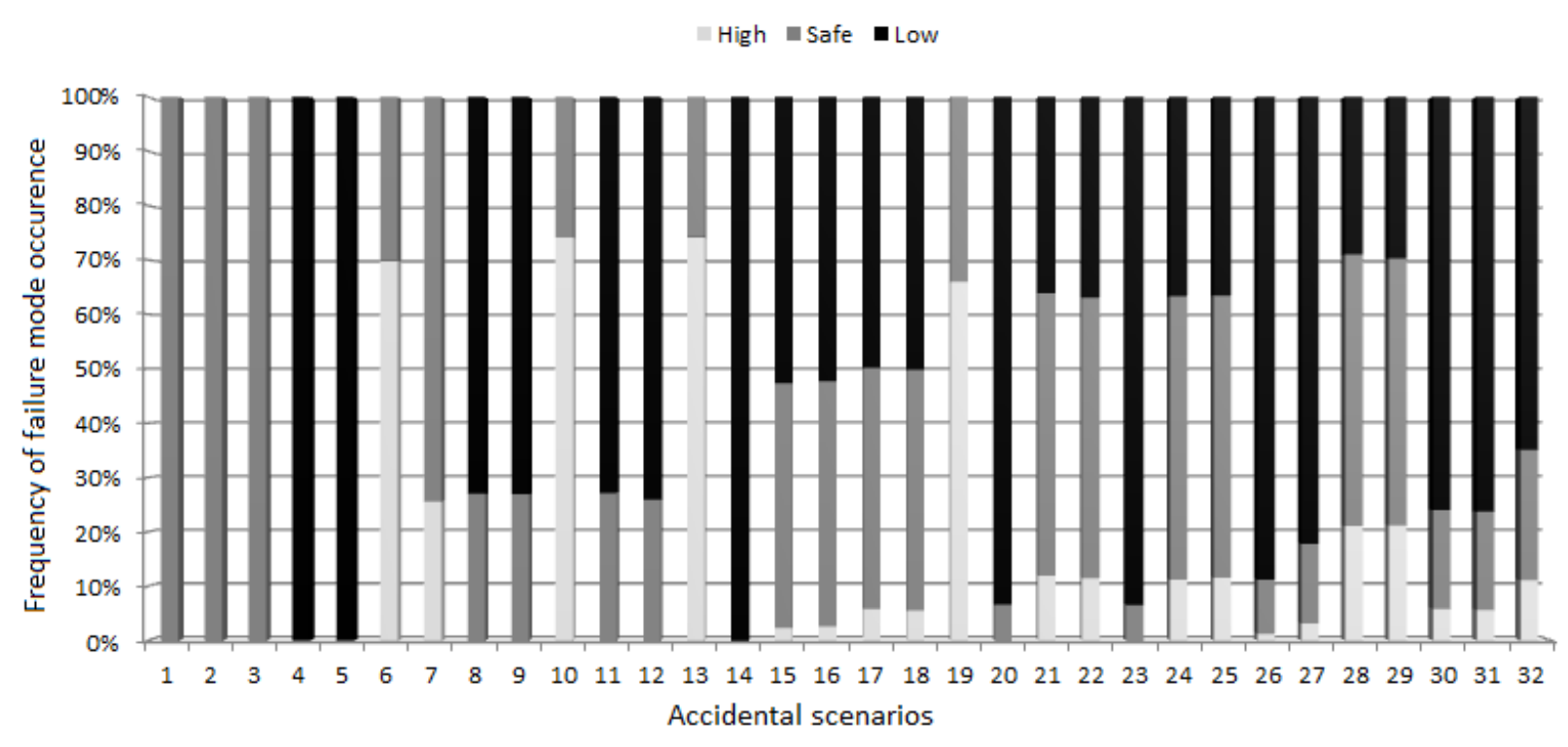

Fig. 3. Histograms of the frequency of the failure modes for each of the 32 system configurations of Tab. 1, simulated with random failure times and magnitudes

Note that non-coherence of the system is maintained: some of the 32 system configurations do not end in the same failure mode all the time. In fact, as shown in Fig. 4 and Fig. 5, both failed and working states of a set of components can contribute to the failure of the system. In Fig. 4 (left) the safety-relevant signal evolution is shown when components $B$ and $D$ continue working for the whole mission time, whereas component $A$ fails at $t=0, C$ at $t=2$ and $E$ at $t=2$ : this combination of failures allows the system to work in "Safe" conditions. On the other hand, in Fig. 4 (upper right) the same signal is plotted when components $B, D$ and $E$ (recovered) continue working for all the mission time, component $A$ fails at $t=0$ and $C$ at $t=2$, leading the system to "Low" failure mode; when components $B, C$ and $D$ (recovered) continue working for all the mission time, component $A$ fails at $t=0$ and $E$ at $t=2$ as in Fig. 4 (lower right), the system failure mode is "High".

Another example of non-coherence is shown in Fig. 5. A combination of three failures allows the system working in "Safe" conditions (left), whereas the recovery of component $C$ leads the system into "High" failure mode (upper right) and the recovery of component $B$ into "Low" (lower right). 


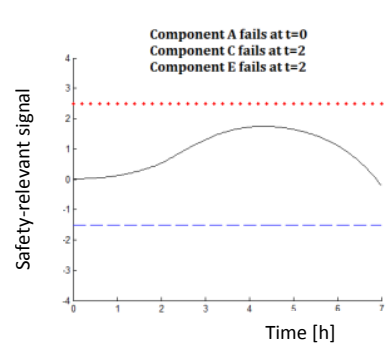

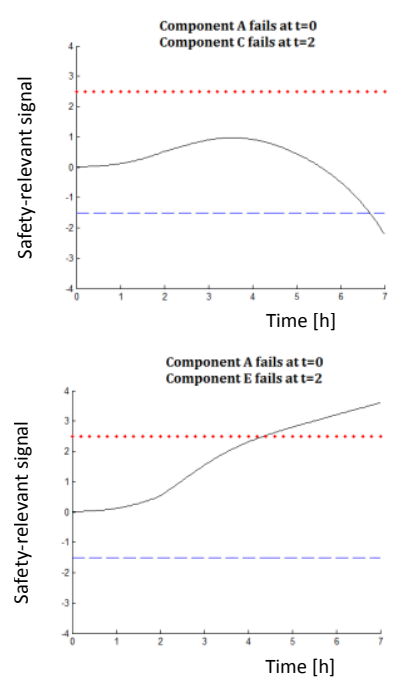

Fig 4. Example of non-coherence
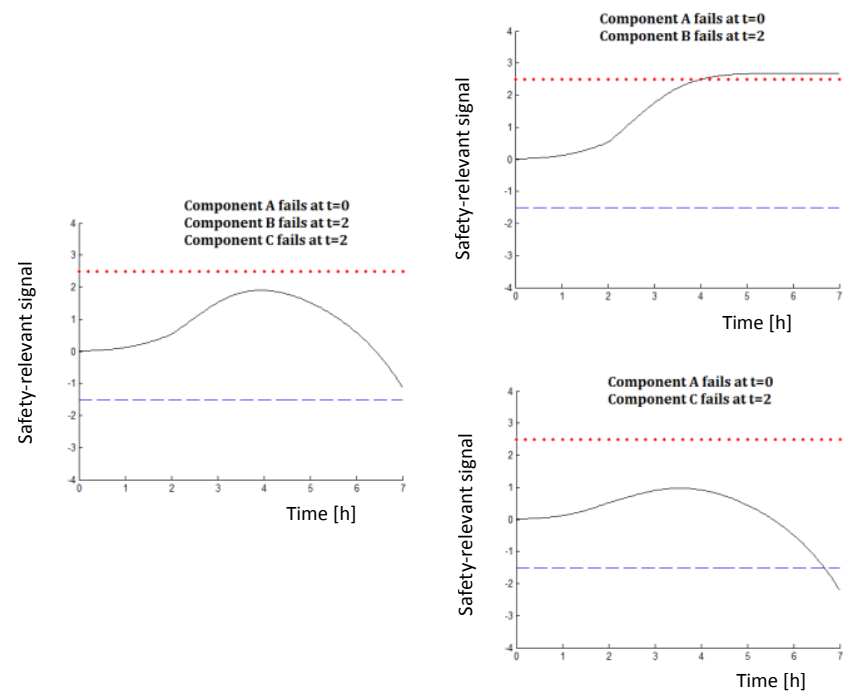

Fig. 5. Example of non-coherence

\section{MULTIPLE-VALUED LOGIC FOR PIs IDENTIFICATION}

In a dynamic and non-coherent system as the one described in Section 2.2, the PIs identification is challenged by the need of representing the components that fail at discrete times ("early", "intermediate" and "late"). For this, we originally adopt a MVL rather than the Boolean logic. In our case, the different value levels of the multistate variables indicate the different time instants at which the components fail. In particular, with respect to component $X$, the multistate variable can assume four different values:

- $\quad X @ t=0$, if $X$ fails at $t=0$ ("early failure")

- $\quad X @ t=2$, if $X$ fails at $t=2$ ("intermediate failure")

- $\quad X @ t=5$, if $X$ fails at $t=5$ ("late failure")

- $\quad \bar{X}$, if $X$ does not fail

The concepts of minterm and PI can be applied to multistate components as well, provided that the variables that represent the components states are MVL rather than Boolean.

As an example, the minterm $|A @ t=2| \bar{B}|C @ t=5| *|E @ t=0|$ stands for an accident scenario where component $B$ continues working, the state of component $D$ is negligible for the determination of the system failure mode ("don't care" value, $|*|$ ), component $A$ fails at $t=2, C$ at $t=5$ and $E$ at $t=0$. Similarly, we can extend the definition of MVL to implicants and PIs. 
Traditionally, within the MVL framework, implicants can be reduced analytically by consensus operation yielding PIs [Ogunbiyi et al., 1981]: reduced implicants are created starting from the whole list of those leading the system to the failure mode of interest. The basic simplifying operation, called merging rule, is easy to apply: if $s$ implicants are the same except for exactly one $s$-th event entry (where $s$ is the number of states that the variables that represent each component can assume, equal to 4 in this case), and all the $s$ possible states of the input variables exist in these implicants, then the $s$ implicants can be merged and form a more reduced implicant [Ogunbiyi et al., 1981]. In Tab. 5, an example of the merging rule is shown: 4 implicants are listed where components $B, C, D$ and $E$ appear in the same state ( $B$ failed at $t=5, C$ failed at $t=0, D$ failed at $t=0$ and $E$ working), while component $A$ appears with a different state in each implicant. Therefore, we can apply the merging rule and obtain the reduced implicant shown on the last row of Tab. 5.

\begin{tabular}{|c|c|c|c|c|}
\hline $\bar{A}$ & $B @ t=5$ & $C @ t=0$ & $D @ t=0$ & $\bar{E}$ \\
\hline$A @ t=0$ & $B @ t=5$ & $C @ t=0$ & $D @ t=0$ & $\bar{E}$ \\
\hline$A @ t=2$ & $B @ t=5$ & $C @ t=0$ & $D @ t=0$ & $\bar{E}$ \\
\hline$A @ t=5$ & $B @ t=5$ & $C @ t=0$ & $D @ t=0$ & $\bar{E}$ \\
\hline- & $B @ t=5$ & $C @ t=0$ & $D @ t=0$ & $\bar{E}$ \\
\hline
\end{tabular}

Tab. 5. Example of an application of the merging rule

The PIs identification method by consensus operation [Ogunbiyi et al., 1981] proceeds iteratively to the reduction of the list of implicants until the whole list of PIs are found. In the case of the system of Section 2.2 with respect to the "Low" failure mode, the consensus method finds the PIs listed in Appendix A. This method is simple to be implemented although it proves to be burdensome when applied to a complex dynamic system. For this reason, we resort to the powerful DE algorithm, whose basic concepts are quickly recalled in the next Section.

\section{DIFFERENTIAL EVOLUTION ALGORITHM}

DE belongs to the class of Evolutionary Algorithms (EAs) [Holland, 1975], which have proven effective in tackling optimization problems with high complexity, number of variables and dimensionality [Storn et al., 1996]. DE search for the optimum entails three phases, called mutation, crossover and selection [Wang et al., 2010]. In the mutation phase, a probability estimation vector $P(\bar{x})=\left[P\left(x_{1}\right), P\left(x_{2}\right), \ldots, P\left(x_{R}\right)\right]$ is created for each chromosome $\bar{x}=\left(x_{1}, x_{2}, \ldots, x_{R}\right)$ present in the population at the $g$-th generation, $g=1,2, \ldots, G$ : 


$$
P\left(x_{r}\right)=\frac{1}{1+e^{-\frac{2 b \cdot\left[x_{r}^{l}+F \cdot\left(x_{r}^{k}-x_{r}^{m}\right)-0.5\right]}{1+2 F}}}
$$

where $b \in[6,9]$ is a positive real constant, the weighting factor $F \in[0,2]$ is a user-defined parameter, kept constant during the optimization and $x_{r}^{l}, x_{r}^{k}$ and $x_{r}^{m}$ are the $r$-th bits of three randomly chosen individuals, with indexes $l, k, m \in\{1,2, \ldots, N P\}$. From $P\left(x_{r}\right)$, the corresponding bit of the noisy vector $\bar{v}$ of the current chromosome $\bar{x}$ are generated by sampling:

$v_{r}= \begin{cases}1 & \text { if } \text { rand } \leq P\left(x_{r}\right) \\ 0 & \text { otherwise }\end{cases}$

where rand is a uniform random number in $[0,1)$.

The trial chromosome $\bar{u}$ can be obtained by the crossover operator through Eq. (3):

$u_{r}= \begin{cases}v_{r} & \text { if } \operatorname{rand} \leq C R \text { or } r=\operatorname{irand}(R) \\ x_{r} & \text { otherwise }\end{cases}$

where $C R \in[0,1]$ is a control parameter which influences the probability for $\bar{v}$ to be selected for the mutation process, $\operatorname{irand}(R)$ is a uniform discrete random number from the set $\{1,2, \ldots, R\}$, where $R$ is the length of the chromosome. Therefore, at least one bit of the trial individual is inherited from the mutant individual so that DE is able to avoid duplication individuals and effectively search within the neighborhood.

During the selection process, the population is modified by substitution. Referring to a minimization search, if the fitness of the trial chromosome $\bar{u}$, i.e., the total cost of all the $\Pi$ belonging to $\bar{u}$, is less than the fitness of the optimal chromosome $\bar{x}$, the former will be a member of the next generation $\mathrm{g}+1$, replacing the target individual, and the trial vector is discarded $\bar{x}= \begin{cases}\bar{u} & \text { if fitness }(\bar{u})<\text { fitness }(\bar{x}) \\ \bar{x} & \text { otherwise }\end{cases}$

It can be understood, then, that the selection criterion of DE is greedy, which guarantees that the following generation is better than or at least equal to the previous generation.

\section{RESULTS}

The results are illustrated for the "Low" failure mode as presented in Section 2.2. The optimization task regards the identification of all minterms (635) and implicants (1166) that lead the system to a "Low" failure mode, coherently with the NP-complete problem of covering a set (the minterms) 
with elements from a given subset (the implicants) [Sen, 1993]: a literal cost is associated to each implicant [Di Maio et al., 2014], equal to the number of components whose MVL state is specified in the implicant, and the PIs are chosen as the implicants subset which cover all the 635 minterms at a minimal literal cost.

The DE algorithm manipulates $R$-dimensional chromosomes $\bar{x}=\left(x_{1}, x_{2}, \ldots, x_{R}\right)$, where $R$ is equal to the number of implicants (equal to 1166 in our case) and a 1 is allocated in the $i$-th position if the $i$ th implicant is chosen to be in the trial solution represented by the chromosome, otherwise a 0 . We apply the DE algorithm with a "One complement" fitness function [Shackleford et al., 2001]: the cost of the solution (chromosome) is mapped into a binary function made up by two parts, where the most important digits are determined as the complement to one of the number of uncovered minterms, whereas the least important digits are determined as the complement to one of the literal cost of the implicants included in the solution. Therefore, we are looking for the solution with the highest fitness value. For the ease of clarity, since we have 635 minterms, 10 bits code the maximum number of uncovered minterms whereas the sum of the cost of all the 1166 implicants is equal to 5320 so that 13 bits code the cost part of the solution. In Fig. 6 the calculation procedure is shown for the solution with the highest fitness value (which corresponds to PIs): the uncovered minterms are equal to zero, while the total cost of the 180 PIs is equal to 661 . The complement to one of 0 on 10 bits is equal to 1023, and the complement to one of 661 on 13 bits is equal to 7350 : joining together the two parts of the fitness function gives a fitness value of 8387946 .

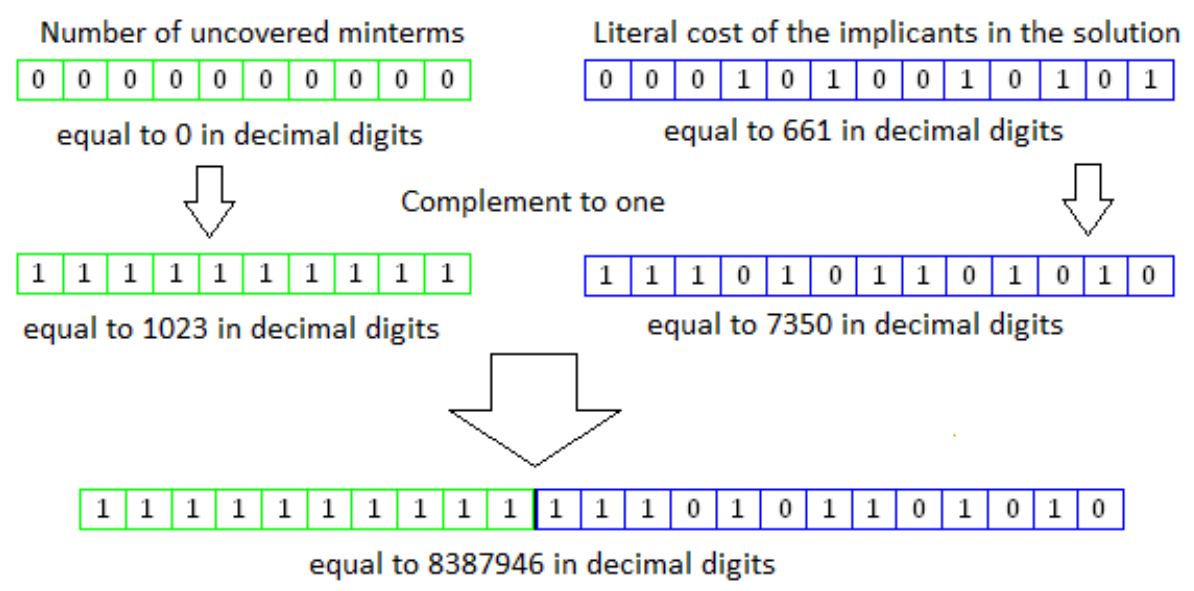

Fig.6. Procedure for the calculation of the "One complement" fitness function

The number of chromosomes, $N P$, is set equal to 5000 and the maximum generation number, MAXGEN, is set equal to 2000. In Fig. 7, Fig. 8, Fig. 9, Fig. 10 and Fig. 11, the fitness values of the 
population in the DE search is plotted at the generation $G=1, G=500, G=1000, G=1500$ and $G=2000$, respectively.

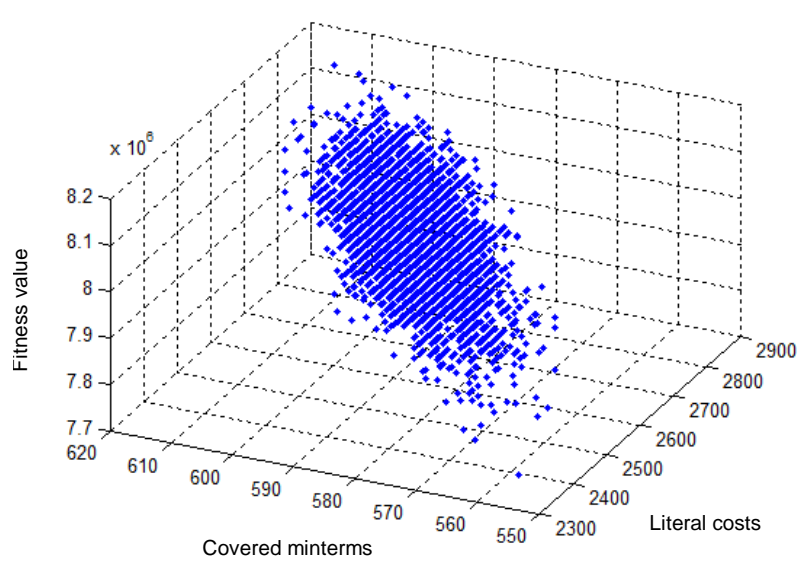

Fig. 7. Fitness values of the population at generation $G=1$

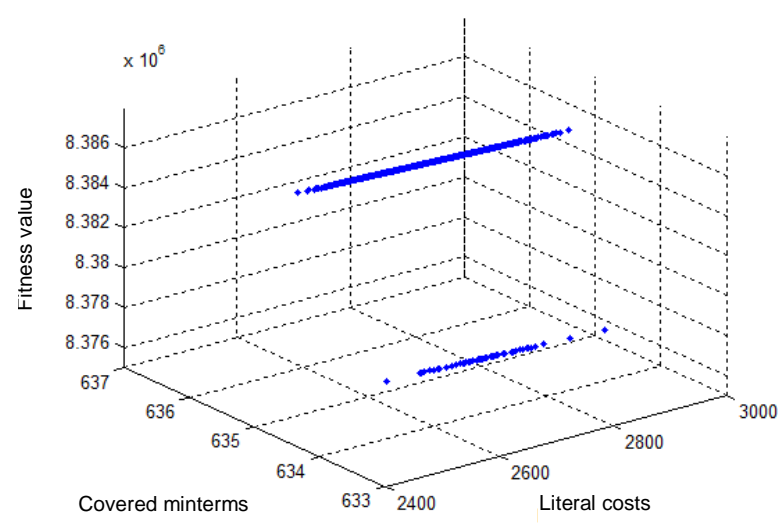

Fig. 8. Fitness values of the population at generation $G=500$

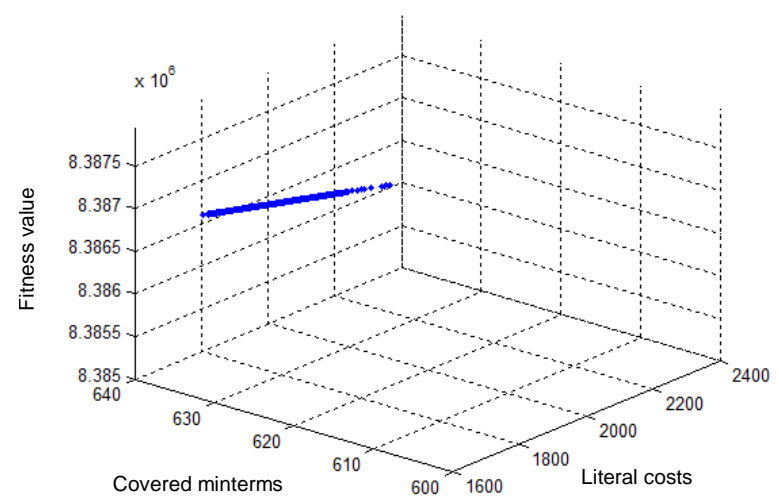

Fig. 9. Fitness values of the population at generation $G=1000$

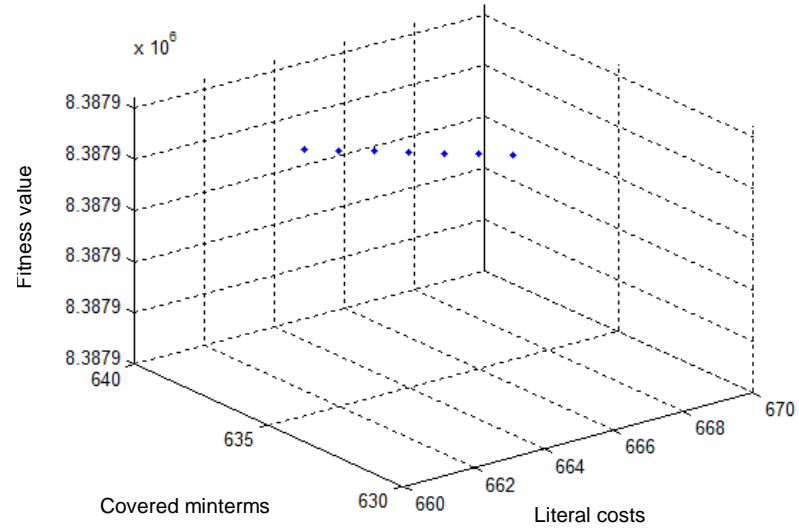

Fig. 10. Fitness values of the population at generation $G=1500$

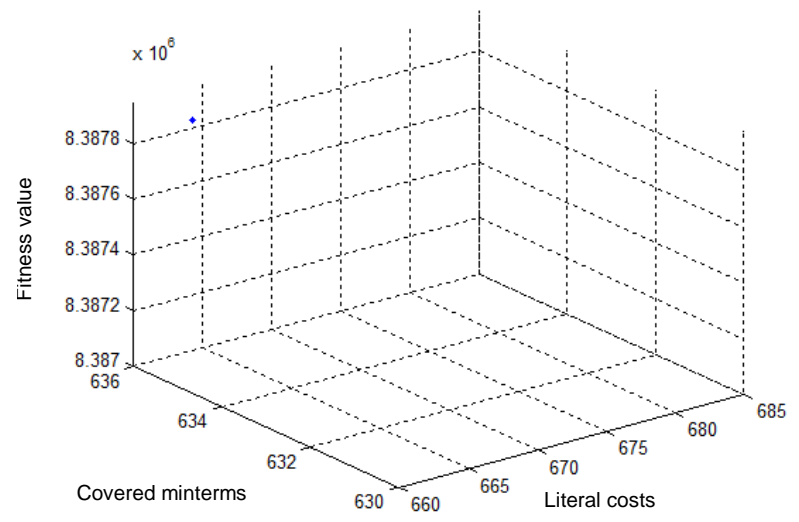

Fig. 11. Fitness values of the best solution obtained by the DE search at generation $G=2000$ 
The population at the first generation (Fig. 8) is randomly distributed in the search space; then, as generations evolve it tends to move toward the optimal solution with largest fitness value (Fig. 11). At the beginning, the population tends to cover all the minterms, even if the solutions have high literal costs (Figs. 9 and 10); then, when all minterms are covered, the DE algorithm moves toward the minimal literal costs (Figs. 10 and 11).

The optimal solution is composed by 180 PIs, which cover all the 635 minterms with literal cost equal to 661. These results are the same as those obtained by the consensus method (Appendix A). A comparison with the PIs listed in Tab. 4 for the static reliability analysis (Section 2.1) highlights that the inclusion of the time of component failures in the definition of PIs leads to a significant increase of the PIs (from 7 to 180). For example, if we look for the subset of the PIs where components $A$ and $E$ do not fail, we find that in the static analysis there are two PIs (rows 1 and 3 of Table 4, respectively):

$$
\begin{aligned}
& \text { - }|\bar{A}| C|\bar{E}| \\
& \text { - } \quad|\bar{A}| D|\bar{E}|
\end{aligned}
$$

whereas among the dynamic PIs we find:

$$
\begin{array}{ll}
- & |\bar{A}| C @ t=0|\bar{E}| \\
- & |\bar{A}| D @ t=0|\bar{E}| \\
- & |\bar{A}| C @ t=2|\bar{E}| \\
- & |\bar{A}| D @ t=2|\bar{E}| \\
- & |\bar{A}| C @ t=5|D @ t=5| \bar{E} \mid \\
- & |\bar{A}| \bar{B}|C @ t=5| \bar{E} \mid \\
- & |\bar{A}| \bar{B}|D @ t=5| \bar{E} \mid \\
\text { - } & |\bar{A}| B @ t=2|C @ t=5| \bar{E} \mid \\
\text { - } & |\bar{A}| B @ t=2|D @ t=5| \bar{E} \mid \\
\text { - } & |\bar{A}| B @ t=5|C @ t=5| \bar{E} \mid \\
\text { - } & |\bar{A}| B @ t=5|D @ t=5| \bar{E} \mid
\end{array}
$$

This shows that a number of implicants (listed below) cannot be considered PIs because in the dynamic analysis these accident scenarios do not lead the system to the "Low" failure mode, although they are included in the set of those covered by rows 1 and 3 of Table 4, herein:

- $|\bar{A}| C @ t=5|\bar{E}|$ 


$$
\begin{array}{ll}
- & |\bar{A}| D @ t=5|\bar{E}| \\
- & |\bar{A}| C @ t=5|\bar{D}| \bar{E} \mid \\
- & |\bar{A}| \bar{C}|D @ t=5| \bar{E} \mid \\
- & |\bar{A}| B @ t=0|C @ t=5| \bar{E} \mid \\
- & |\bar{A}| B @ t=0|D @ t=5| \bar{E} \mid \\
- & |\bar{A}| B @ t=0|C @ t=5| \bar{D}|\bar{E}| \\
- & |\bar{A}| B @ t=0|\bar{C}| D @ t=5|\bar{E}|
\end{array}
$$

\section{CONCLUSIONS}

In the risk analysis of dynamic systems, timing of the events influences the development of the accident scenario, and, in case of non-coherent systems both failed and working states of the same components can lead to system failure. Traditional MCSs lose their meaning in these cases, and for this reason, PIs have been introduced as an extension of MCSs to convey the information on the minimum combinations of failures (with a certain order and timing) that lead the system to failure. In this paper, we have presented an innovative framework for PIs identification in dynamic noncoherent systems. The proposed framework relies on MVL theory to account for the timing of the component failures in the definition of the accident scenarios and on a DE-based optimization algorithm for efficiently identifying the PIs. A case study has been solved by the proposed framework and the results have been compared to those obtained by a Quine-McCluskey algorithm and consensus operation method. The proposed framework has been shown faster than QuineMcCluskey and consensus operation method, and also the results have confirmed the importance of including the dynamic aspects in the reliability analysis of systems for which the timing of events is relevant for the accident scenario evolution: indeed, in the example shown, some of the MCSs identified cannot be considered mimum combinations of failures leading to system failure, whereas PIs are capable of properly capturing the mimimum failure conditions.

\section{Acknowledgements}

The participation of Enrico Zio to this research is partially supported by the China NSFC under grant number 71231001. 


\section{References}

[Aldemir, 2013] Aldemir T., "A survey of dynamic methodologies for probabilistic safety assessment of nuclear power plant”, Annals of Nuclear Energy, Volume 52, pp.113-124, 2013.

[Baraldi et al., 2012] Baraldi P., Di Maio F., Zio E., "Unsupervised Clustering for Fault Diagnosis", Proceedings of Prognostics and System Health Management Conference (PHM-2012), Beijing, China, 23-25 May 2012.

[Baraldi et al., 2013] Baraldi P., Di Maio F., Zio E., "Unsupervised Clustering for Fault Diagnosis in Nuclear Power Plant Components", International Journal of Computational Intelligence Systems, Vol. 6, No. 4, pp. 764-777, 2013.

[Beasley et al., 1996] Beasley J.E., Chu P.C., “A genetic algorithm for the set covering problem”, European Journal of Operational Research, vol.94, pp. 392-404, 1996.

[Devooght et al., 1992] Devooght D., Smidts C., "Probabilistic reactor dynamics I: the theory of continuous event trees", Nuclear Science and Engineering, Volume 111, pp.229-240, 1992.

[Devooght, 1997] Devooght D., "Dynamic reliability", Advances in Nuclear Science and Technology, Volume 25, pp.215-278, 1997.

[Di Maio et al., 2011a] Di Maio F., Secchi P., Vantini S., Zio E., "Fuzzy C-Means Clustering of Signal Functional Principal Components for Post-Processing Dynamic Scenarios of a Nuclear Power Plant Digital Instrumentation and Control System", IEEE Transactions on Reliability, pp.415-425, 2011.

[Di Maio et al., 2011b] Di Maio F., Zio E., Jiejuan Tong T.L., "Passive system accident scenario analysis by simulation", ANS PSA 2011 International Topical Meeting on Probabilistic Safety Assessment and Analysis, Wilmington, NC, March 13-17, 2011.

[Di Maio et al., 2013] Di Maio F., Baronchelli S., Zio E., "Minimal Cut Sets Identification by Hierarchical Differential Evolution", PSA 2013, the International Topical Meeting on Probabilistic Safety Assessment and Analysis, 22-27 September 2013, Columbia, South Carolina, USA.

[Di Maio et al., 2014] F. Di Maio, S. Baronchelli, E. Zio, "Hierarchical Differential Evolution for Minimal Cut Sets Identification: Application to Nuclear Safety Systems", European Journal of Operational Research, Volume 238, Issue 2, Pages 645-652, 2014.

[Garibba et al., 1985] Garibba S., Guagnini E., Mussio P., “Multiple-Valued Logic Trees: Meaning and Prime Implicants”, IEEE Transactions on Reliability, Volume R-34, No. 5, pp.463-472, 1985 [Garret et al., 1999] Garret C. J., Apostolakis G.E., "Context in the risk assessment of digital systems", Risk Analysis, Volume 19, Issue 1, February 1999, Pages 23-32. 
[Hakobyan et al., 2008] Hakobyan A., Denning R., Aldemir T., Dunagan S., Kunsman D., “ $A$ Methodology for Generating Dynamic Accident Progression Event Trees for Level 2 PRA". SAND2008-4746, Sandia National Laboratories Albuquerque, New Mexico, 2008.

[Hofer et al., 2004] Hofer E., Kloos M., Krzykacz-Hausmann B., Peschke J., Sonnenkalb M., “Dynamic Event Trees for Probabilistic Safety Analysis”, GRS, Garsching, Germany.

[Holland, 1975] Holland J.H., "Adaptation in Natural and Artificial Systems", University of Michigan Press, Ann Arbor, USA, 1975.

[Kirschenbaum et al., 2009] Kirschenbaum J., Bucci P., Stovsky M., Mandelli D., Aldemir T., Yau M., Guarro S., Ekici E., Arndt S.A., "A benchmark system for comparing reliability modeling approaches for digital instrumentation and control systems", Nuclear Technology, Volume 165, pp.53-95, 2009.

[Kopustinskas et al., 2005] Kopustinskas V., Augutis J., Rimkevicius S., "Dynamic reliability and risk assessment of the accident localization system of the Ignalina NPP RBMK-1500 reactor", Reliability Engineering and System Safety, Volume 87, pp.77-87, 2005.

[Labeau et al., 2000] Labeau P.E., Smidts C., Swaminathan S., "Dynamic reliability: towards an integrated platform for probabilistic risk assessment”, Reliability Engineering and System Safety, Volume 68, pp.219-254, 2000.

[Labeau, 2006] Labeau, P.E., “A survey on Monte Carlo estimation of small failure risks in dynamic reliability”, International Journal of Electronics and Communication, Volume 52, pp.205-211, 2006.

[Li et al., 2005] Bin Li, Ming Li, Carol Smidts, "Integrating Software into PRA: A Test-Based Approach”, Risk Analysis, Vol. 25, No. 4, 2005.

[Marseguerra et al., 1996] Marseguerra M., Zio E., "Monte Carlo approach to PSA for dynamic process system”, Reliability Engineering and System Safety, Volume 52, pp.227-241, 1996.

[Morreale, 1967] Morreale E., "Partitioned List Algorithms for Prime Implicant Determination from Canonical forms", IEEE Transactions on Electronic Computers, Volume EC-16, No.5, 611620, 1967.

[Ogunbiyi et al., 1981] Ogunbiyi E.I., Henley E.J., "Irredundant Forms and Prime Implicants of a Function with Multistate Variables", IEEE Transactions on Reliability, Volume R-30, No. 1, pp.3942, 1981.

[Pate'-Cornell, 2002] Pate'-Cornell, E., "Finding and Fixing Systems Weaknesses: Probabilistic Methods and Applications of Engineering Risk Analysis", Risk Analysis, Vol. 22, No. 2, 2002.

[Petersen, 1981] Petersen J.L., "Petri net theory and the modeling of systems", Englewood Cliffs, NJ: Prentice-Hall, 1981. 
[Quine, 1952] Quine W.V., “The problem of simplifying truth functions”, Am. Math. Monthly, Volume 59, 521-531, 1952.

[Rosenberg, 1996] Rosenberg L., "Algorithm for finding minimal cut sets in a fault tree”, Reliability Engineering and System Safety 53, 67-71, 1996.

[Sen, 1993] Sen S., "Minimal cost set covering using probabilistic methods", Proceedings of the 1993 ACM/SIGAPP symposium on Applied computing: states of the art and practice, 157-164, 1993.

[Shackleford et al., 2001] Shackleford B., Snider G., Carter R.J., Okushi E., Yasuda M., Seo K., Yasuura H., "A High-Performance, Pipelined, FPGA-Based Genetic Algorithm Machine", Genetic Programming and Evolvable Machines, Volume 2, Number 1, 33-60, 2001.

[Sharvia et al., 2008] Sharvia S., Papadopoulos, "Non-coherent Modelling in Compositional Fault Tree Analysis", Proceedings of the 17th World Congress, The International Federation of Automatic Control, Seoul, Korea, July 6-11, 2008.

[Stuk, 1993] STUK, "Probabilistic Safety Analysis in Safety Management of Nuclear Power Plants", Finnish Radiation and Nuclear Safety Authority (STUK) Guide, YVL-2.8, 1993.

[Storn et al., 1996] Storn R., Price K., "Differential Evolution - a simple and efficient heuristic for global optimization over continuous spaces", Journal of Global Optimization, Volume 11, pp.341359, 1996.

[Wang et al., 2010] Wang L., Fu X., Menhas M.I., “A Modified Binary Differential Evolution Algorithm", Life Modelling and Intelligent Computing, Lectures Notes in Computer Science, Volume 6329, pp.49-57, 2010.

[Zio, 2007] Zio E., "An Introduction to the Basics of Reliability and Risk Analysis", World Scientific Publishing Company, Series on Quality, Reliability and Engineering Statistics, Vol 13, ISBN: 9812706399, 2007

[Zio et al. 2009] Zio E., Di Maio F., "Processing Dynamic Scenarios from a Reliability Analysis of a Nuclear Power Plant Digital Instrumentation and Control System", Annals of Nuclear Energy, Volume 36, pp.1386-1399, 2009.

[Zio et al., 2010] E. Zio, F. Di Maio, J. Tong, "Safety Margins Confidence Estimation for a Passive Residual Heat Removal System”, Reliability Engineering and System Safety, RESS, Vol. 95, 2010, pp. 828-836, doi:10.1016/j.ress.2010.03.006, 2010.

[Zio et al., 2012] Zio E., Di Maio F., "Fault Diagnosis and Failure Mode Estimation Data-Driven Fuzzy Similarity Approach", International Journal of Performability Engineering, Volume 8, No. 1, pp.49-65, 2012. 


\section{APPENDIX A}

List of PIs for the system with discrete times of failure events ("early", "intermediate" and "late", $t=0,2,5$, respectively)

\section{Prime Implicants (Dynamic Reliability Analysis)}

\begin{tabular}{|c|c|c|c|}
\hline 1. & $|C @ t=0| D @ t=0|\bar{E}|$ & 91. & $|\bar{A}| B @ t=5|D @ t=2| E @ t=2 \mid$ \\
\hline 2. & $|C @ t=0| D @ t=0|E @ t=5|$ & 92. & $|\bar{A}| B @ t=5|D @ t=2| E @ t=5 \mid$ \\
\hline 3. & $|C @ t=0| D @ t=0 \mid E @ t=2$ & 93. & $|\bar{A}| B @ t=5|D @ t=5| \bar{E} \mid$ \\
\hline 4. & $|C @ t=0| D @ t=2|\bar{E}|$ & 94. & $|\bar{A}| B @ t=5|D @ t=5| E @ t=5 \mid$ \\
\hline 5. & $|C @ t=0| D @ t=2|E @ t=5|$ & 95. & $|\bar{A}| B @ t=5|C @ t=0| E @ t=2 \mid$ \\
\hline 6. & $|C @ t=0| D @ t=2 \mid E @ t=2$ & 96. & $|\bar{A}| B @ t=5|C @ t=2| E @ t=2 \mid$ \\
\hline 7. & $|C @ t=0| D @ t=5|\bar{E}|$ & 97. & $|\bar{A}| B @ t=5|C @ t=0| E @ t=5 \mid$ \\
\hline 8. & $|C @ t=0| D @ t=5|E @ t=5|$ & 98. & $|\bar{A}| B @ t=5|C @ t=2| D @ t=5 \mid$ \\
\hline 9. & $|C @ t=2| D @ t=0|\bar{E}|$ & 99. & $|\bar{A}| B @ t=5|C @ t=5| \bar{E} \mid$ \\
\hline 10. & $|C @ t=2| D @ t=0|E @ t=5|$ & 100. & $|\bar{A}| B @ t=5|C @ t=5| E @ t=5 \mid$ \\
\hline 11. & $|C @ t=2| D @ t=0 \mid E @ t=2$ & 101. & $|\bar{A}| B @ t=5|C @ t=5| D @ t=2 \mid$ \\
\hline 12. & $|C @ t=2| D @ t=2|\bar{E}|$ & 102. & $|\bar{A}| B @ t=5|C @ t=5| D @ t=5 \mid$ \\
\hline 13. & $|C @ t=2| D @ t=2|E @ t=5|$ & 103. & $|A @ t=2| C @ t=0|D @ t=0|$ \\
\hline 14. & $|C @ t=2| D @ t=5|\bar{E}|$ & 104. & $|A @ t=2| C @ t=2|D @ t=2| E @ t=2 \mid$ \\
\hline 15. & $|C @ t=2| D @ t=5|E @ t=5|$ & 105. & $|A @ t=2| \bar{B}|D @ t=2| E @ t=5 \mid$ \\
\hline 16. & $|C @ t=5| D @ t=0|\bar{E}|$ & 106. & $|A @ t=2| \bar{B}|D @ t=5| \bar{E} \mid$ \\
\hline 17. & $|C @ t=5| D @ t=0|E @ t=5|$ & 107. & $|A @ t=2| \bar{B}|C @ t=2| \bar{E} @ t=5 \mid$ \\
\hline 18. & $|C @ t=5| D @ t=2|\bar{E}|$ & 108. & $|A @ t=2| \bar{B}|C @ t=2| D @ t=5 \mid$ \\
\hline 19. & $|C @ t=5| D @ t=2|E @ t=5|$ & 109. & $|A @ t=2| \bar{B}|C @ t=5| \bar{E} \mid$ \\
\hline 20. & $|\bar{B}| D @ t=0|\bar{E}|$ & 110. & $|A @ t=2| \bar{B}|C @ t=5| D @ t=2 \mid$ \\
\hline 21. & $|\bar{B}| D @ t=0|E @ t=5|$ & 111. & $|A @ t=2| \bar{B}|C @ t=5| D @ t=5|E @ t=2|$ \\
\hline 22. & $|\bar{B}| D @ t=2|\bar{E}|$ & 112. & $|A @ t=2| B @ t=2|D @ t=0| \bar{E} \mid$ \\
\hline 23. & $|\bar{B}| C @ t=0|\bar{E}|$ & 113. & $|A @ t=2| B @ t=2|D @ t=2| \bar{E} \mid$ \\
\hline 24. & $|\bar{B}| C @ t=0|E @ t=5|$ & 114. & $|A @ t=2| B @ t=2|C @ t=0| \bar{E} \mid$ \\
\hline
\end{tabular}




\begin{tabular}{|c|c|c|c|}
\hline 25. & $|\bar{B}| C @ t=0|D @ t=0|$ & 115. & $|A @ t=2| B @ t=2|C @ t=0| D @ t=2 \mid$ \\
\hline 26. & $|\bar{B}| C @ t=0|D @ t=2|$ & 116. & $|A @ t=2| B @ t=2|C @ t=0| D @ t=5|E @ t=2|$ \\
\hline 27. & $|\bar{B}| C @ t=0|D @ t=5|$ & 117. & $|A @ t=2| B @ t=2|C @ t=2| \bar{E} \mid$ \\
\hline 28. & $|\bar{B}| C @ t=2|\bar{E}|$ & 118. & $|A @ t=2| B @ t=2|C @ t=2| D @ t=0 \mid$ \\
\hline 29. & $|\bar{B}| C @ t=2|D @ t=0|$ & 119. & $|A @ t=2| B @ t=2|C @ t=2| D @ t=2 \mid$ \\
\hline 30. & $|\bar{B}| C @ t=2|D @ t=2|$ & 120. & $|A @ t=2| B @ t=2|C @ t=2| D @ t=5|E @ t=2|$ \\
\hline 31. & $|\bar{B}| C @ t=2|D @ t=5| E @ t=2 \mid$ & 121. & $|A @ t=2| B @ t=2|C @ t=5| D @ t=0|E @ t=2|$ \\
\hline 32. & $|\bar{B}| C @ t=5|D @ t=0|$ & 122. & $|A @ t=2| B @ t=2|C @ t=5| D @ t=2|E @ t=2|$ \\
\hline 33. & $|\bar{B}| C @ t=5|D @ t=2| E @ t=2 \mid$ & 123. & $|A @ t=2| B @ t=2|C @ t=5| D @ t=5|\bar{E}|$ \\
\hline 34. & $|\bar{B}| C @ t=5|D @ t=5| \bar{E} \mid$ & 124. & $|A @ t=2| B @ t=2|C @ t=5| D @ t=5|E @ t=5|$ \\
\hline 35. & $|\bar{B}| C @ t=5|D @ t=5| E @ t=5 \mid$ & 125. & $|A @ t=2| B @ t=5|D @ t=2| E @ t=5 \mid$ \\
\hline 36. & $|B @ t=2| C @ t=0|D @ t=0|$ & 126. & $|A @ t=2| B @ t=5|C @ t=2| E @ t=5 \mid$ \\
\hline 37. & $|A @ t=2| B @ t=5|D @ t=5| \bar{E} \mid$ & 127. & $|B @ t=2| C @ t=2|D @ t=2| E @ t=2 \mid$ \\
\hline 38. & $|B @ t=5| D @ t=0|\bar{E}|$ & 128. & $|A @ t=2| B @ t=5|C @ t=2| D @ t=5 \mid$ \\
\hline 39. & $|B @ t=5| D @ t=0|E @ t=5|$ & 129. & $|A @ t=2| B @ t=5|C @ t=5| \bar{E} \mid$ \\
\hline 40. & $|B @ t=5| D @ t=2|\bar{E}|$ & 130. & $|A @ t=2| B @ t=5|C @ t=5| D @ t=2 \mid$ \\
\hline 41. & $|B @ t=5| C @ t=0|\bar{E}|$ & 131. & $|A @ t=2| B @ t=5|C @ t=5| D @ t=5|E @ t=2|$ \\
\hline 42. & $|B @ t=5| C @ t=0|E @ t=5|$ & 132. & $|A @ t=5| D @ t=0|\bar{E}|$ \\
\hline 43. & $|B @ t=5| C @ t=0|D @ t=0|$ & 133. & $|A @ t=5| D @ t=0|E @ t=5|$ \\
\hline 44. & $|B @ t=5| C @ t=0|D @ t=2|$ & 134. & $|A @ t=5| D @ t=2|\bar{E}|$ \\
\hline 45. & $|B @ t=5| C @ t=0|D @ t=5|$ & 135. & $|A @ t=5| C @ t=0|\bar{E}|$ \\
\hline 46. & $|B @ t=5| C @ t=2|\bar{E}|$ & 136. & $|A @ t=5| C @ t=0|E @ t=5|$ \\
\hline 47. & $|B @ t=5| C @ t=2|D @ t=0|$ & 137. & $|A @ t=5| C @ t=0|D @ t=0|$ \\
\hline 48. & $|B @ t=5| C @ t=2|D @ t=2|$ & 138. & $|A @ t=5| C @ t=0|D @ t=2|$ \\
\hline 49. & $|A @ t=5| C @ t=0|D @ t=5|$ & 139. & $|B @ t=5| C @ t=2|D @ t=5| E @ t=2 \mid$ \\
\hline 50. & $|B @ t=5| C @ t=5|D @ t=0|$ & 140. & $|A @ t=5| C @ t=2|\bar{E}|$ \\
\hline 51. & $|A @ t=5| C @ t=2|D @ t=0|$ & 141. & $|B @ t=5| C @ t=5|D @ t=2| E @ t=2 \mid$ \\
\hline 52. & $|B @ t=5| C @ t=5|D @ t=5| \bar{E} \mid$ & 142. & $|A @ t=5| C @ t=2|D @ t=5| E @ t=2 \mid$ \\
\hline 53. & $|A @ t=5| C @ t=2|D @ t=2|$ & 143. & $|B @ t=5| C @ t=5|D @ t=5| E @ t=5 \mid$ \\
\hline 54. & $|\bar{A}| D @ t=0|\bar{E}|$ & 144. & $|A @ t=5| C @ t=5|D @ t=0|$ \\
\hline 55. & $|\bar{A}| D @ t=0|E @ t=5|$ & 145. & $|A @ t=5| C @ t=5|D @ t=2| E @ t=2 \mid$ \\
\hline 56. & $|\bar{A}| D @ t=2|\bar{E}|$ & 146. & $|A @ t=5| C @ t=5|D @ t=5| \bar{E} \mid$ \\
\hline 57. & $|\bar{A}| C @ t=0|\bar{E}|$ & 147. & $|A @ t=5| C @ t=5|D @ t=5| E @ t=5 \mid$ \\
\hline 58. & $|\bar{A}| C @ t=0|E @ t=5|$ & 148. & $|A @ t=5| \bar{B}|D @ t=0| E @ t=2 \mid$ \\
\hline 59. & $|\bar{A}| C @ t=0|D @ t=0|$ & 149. & $|A @ t=5| \bar{B}|D @ t=2| E @ t=2 \mid$ \\
\hline 60. & $|\bar{A}| C @ t=0|D @ t=2|$ & 150. & $|A @ t=5| \bar{B}|D @ t=2| E @ t=5 \mid$ \\
\hline 61. & $|\bar{A}| C @ t=0|D @ t=5|$ & 151. & $|A @ t=5| \bar{B}|D @ t=5| \bar{E} \mid$ \\
\hline 62. & $|\bar{A}| C @ t=2|\bar{E}|$ & 152. & $|A @ t=5| \bar{B}|D @ t=5| E @ t=5 \mid$ \\
\hline
\end{tabular}




\begin{tabular}{|c|c|c|c|}
\hline 63. & $|\bar{A}| C @ t=2|D @ t=0|$ & 153. & $|A @ t=5| \bar{B}|C @ t=0| E @ t=2 \mid$ \\
\hline 64. & $|\bar{A}| C @ t=2|D @ t=2|$ & 154. & $|A @ t=5| \bar{B}|C @ t=2| E @ t=2 \mid$ \\
\hline 65. & $|\bar{A}| C @ t=2|D @ t=5| E @ t=2 \mid$ & 155. & $|A @ t=5| \bar{B}|C @ t=2| E @ t=5 \mid$ \\
\hline 66. & $|\bar{A}| C @ t=5|D @ t=0|$ & 156. & $|A @ t=5| \bar{B}|C @ t=2| D @ t=5 \mid$ \\
\hline 67. & $|\bar{A}| C @ t=5|D @ t=2| E @ t=2 \mid$ & 157. & $|A @ t=5| \bar{B}|C @ t=5| \bar{E} \mid$ \\
\hline 68. & $|\bar{A}| C @ t=5|D @ t=5| \bar{E} \mid$ & 158. & $|A @ t=5| \bar{B}|C @ t=5| E @ t=5 \mid$ \\
\hline 69. & $|\bar{A}| C @ t=5|D @ t=5| E @ t=5 \mid$ & 159. & $|A @ t=5| \bar{B}|C @ t=5| D @ t=2 \mid$ \\
\hline 70. & $|\bar{A}| \bar{B}|D @ t=0| E @ t=2 \mid$ & 160. & $|A @ t=5| \bar{B}|C @ t=5| D @ t=5 \mid$ \\
\hline 71. & $|\bar{A}| \bar{B}|D @ t=2| E @ t=2 \mid$ & 161. & $|A @ t=5| B @ t=2|D @ t=2| E @ t=5 \mid$ \\
\hline 72. & $|\bar{A}| \bar{B}|D @ t=2| E @ t=5 \mid$ & 162. & $|A @ t=5| B @ t=2|D @ t=5| \bar{E} \mid$ \\
\hline 73. & $|\bar{A}| \bar{B}|D @ t=5| \bar{E} \mid$ & 163. & $|A @ t=5| B @ t=2|C @ t=2| E @ t=5 \mid$ \\
\hline 74. & $|\bar{A}| \bar{B}|D @ t=5| E @ t=5 \mid$ & 164. & $|A @ t=5| B @ t=2|C @ t=2| D @ t=5 \mid$ \\
\hline 75. & $|\bar{A}| \bar{B}|C @ t=0| E @ t=2 \mid$ & 165. & $|A @ t=5| B @ t=2|C @ t=5| \bar{E} \mid$ \\
\hline 76. & $|\bar{A}| \bar{B}|C @ t=2| E @ t=2 \mid$ & 166. & $|A @ t=5| B @ t=2|C @ t=5| D @ t=2 \mid$ \\
\hline 77. & $|\bar{A}| \bar{B}|C @ t=2| E @ t=5 \mid$ & 167. & $|A @ t=5| B @ t=2|C @ t=5| D @ t=5|E @ t=2|$ \\
\hline 78. & $|\bar{A}| \bar{B}|C @ t=2| D @ t=5 \mid$ & 168. & $|A @ t=5| B @ t=5|D @ t=0| E @ t=2 \mid$ \\
\hline 79. & $|\bar{A}| \bar{B}|C @ t=5| \bar{E} \mid$ & 169. & $|A @ t=5| B @ t=5|D @ t=2| E @ t=2 \mid$ \\
\hline 80. & $|\bar{A}| \bar{B}|C @ t=5| E @ t=5 \mid$ & 170. & $|A @ t=5| B @ t=5|D @ t=2| E @ t=5 \mid$ \\
\hline 81. & $|\bar{A}| \bar{B}|C @ t=5| D @ t=2 \mid$ & 171. & $|A @ t=5| B @ t=5|D @ t=5| \bar{E} \mid$ \\
\hline 82. & $|\bar{A}| \bar{B}|C @ t=5| D @ t=5 \mid$ & 172. & $|A @ t=5| B @ t=5|D @ t=5| E @ t=5 \mid$ \\
\hline 83. & $|\bar{A}| B @ t=2|D @ t=2| E @ t=5 \mid$ & 173. & $|A @ t=5| B @ t=5|C @ t=0| E @ t=2 \mid$ \\
\hline 84. & $|\bar{A}| B @ t=2|D @ t=5| \bar{E} \mid$ & 174. & $|A @ t=5| B @ t=5|C @ t=2| E @ t=2 \mid$ \\
\hline 85. & $|\bar{A}| B @ t=2|C @ t=2| E @ t=5 \mid$ & 175. & $|A @ t=5| B @ t=5|C @ t=2| E @ t=5 \mid$ \\
\hline 86. & $|\bar{A}| B @ t=2|C @ t=2| D @ t=5 \mid$ & 176. & $|A @ t=5| B @ t=5|C @ t=2| D @ t=5 \mid$ \\
\hline 87. & $|\bar{A}| B @ t=2|C @ t=5| \bar{E} \mid$ & 177. & $|A @ t=5| B @ t=5|C @ t=5| D @ t=5 \mid$ \\
\hline 88. & $|\bar{A}| B @ t=2|C @ t=5| D @ t=2 \mid$ & 178. & $|A @ t=5| B @ t=5|C @ t=2| E @ t=5 \mid$ \\
\hline 89. & $|A @ t=5| B @ t=5|C @ t=5| \bar{E} \mid$ & 179. & $|\bar{A}| B @ t=2|C @ t=5| D @ t=5|E @ t=2|$ \\
\hline 90. & $|\bar{A}| B @ t=5|D @ t=0| E @ t=2 \mid$ & 180. & $|A @ t=5| B @ t=5|C @ t=5| D @ t=2 \mid$ \\
\hline
\end{tabular}

